\title{
Higgs transition from a magnetic Coulomb liquid to a ferromagnet in $\mathrm{Yb}_{2} \mathrm{Ti}_{2} \mathrm{O}_{7}$
}

\author{
Lieh-Jeng Chang ${ }^{1,2}$, Shigeki Onoda ${ }^{3}$, Yixi Su${ }^{4}$, Ying-Jer Kao ${ }^{5}$, Ku-Ding Tsuei $^{6}$, Yukio Yasui ${ }^{7,8}$, \\ Kazuhisa Kakurai ${ }^{2} \&$ Martin Richard Lees ${ }^{9}$
}

In a class of frustrated magnets known as spin ice, magnetic monopoles emerge as classical defects and interact via the magnetic Coulomb law. With quantum-mechanical interactions, these magnetic charges are carried by fractionalized bosonic quasi-particles, spinons, which can undergo Bose-Einstein condensation through a first-order transition via the Higgs mechanism. Here, we report evidence of a Higgs transition from a magnetic Coulomb liquid to a ferromagnet in single-crystal $\mathrm{Yb}_{2} \mathrm{Ti}_{2} \mathrm{O}_{7}$. Polarized neutron scattering experiments show that the diffuse [111]-rod scattering and pinch-point features, which develop on cooling are suddenly suppressed below $T_{C} \sim 0.21 \mathrm{~K}$, where magnetic Bragg peaks and a full depolarization of the neutron spins are observed with thermal hysteresis, indicating a first-order ferromagnetic transition. Our results are explained on the basis of a quantum spin-ice model, whose hightemperature phase is effectively described as a magnetic Coulomb liquid, whereas the ground state shows a nearly collinear ferromagnetism with gapped spin excitations.

\footnotetext{
${ }^{1}$ Department of Physics, National Cheng Kung University, Tainan 70101, Taiwan. ${ }^{2}$ Quantum Beam Science Directorate, Japan Atomic Energy Agency, Tokai, Ibaraki 319-1195, Japan. ${ }^{3}$ Condensed Matter Theory Laboratory, RIKEN, Wako, Saitama 351-0198, Japan. ${ }^{4}$ Jülich Centre for Neutron Science JCNSFRM II, Forschungszentrum Jülich GmbH, Outstation at FRM II, Lichtenbergstrasse 1, D-85747 Garching, Germany. ${ }^{5}$ Department of Physics and Center for Advanced Study in Theoretical Science, National Taiwan University, Taipei 10607, Taiwan. 6 National Synchrotron Radiation Research Center, Hsinchu 30076, Taiwan. ${ }^{7}$ Department of Physics, Division of Material Science, Nagoya University, Furo-cho, Chikusa-ku, Nagoya 464-8602, Japan. ${ }^{8}$ Department of Physics, Meiji University, Kawasaki 214-8571, Japan. ${ }^{9}$ Department of Physics, University of Warwick, Coventry CV4 7AL, UK. Correspondence and requests for materials should be addressed to L.J.C. (email: Ijchang@mail.ncku.edu.tw) or to S.O. (email: s.onoda@riken.jp).
} 
E mergent magnetic monopoles ${ }^{1}$ have recently been identified in many condensed-matter systems ${ }^{2-5}$. In particular, recent experiments ${ }^{6-9}$ have shown that the low-temperature state of dipolar spin-ice materials ${ }^{10,11}, \mathrm{Ho}_{2} \mathrm{Ti}_{2} \mathrm{O}_{7}$ and $\mathrm{Dy}_{2} \mathrm{Ti}_{2} \mathrm{O}_{7}$, can be effectively described as a classical magnetic Coulomb phase, which hosts these emergent magnetic monopoles obeying the magnetic Coulomb law ${ }^{2,3}$. Theoretically, this has been understood from a classical model where $\langle 111\rangle$ Ising moments, that is, pseudospin-1/2 moments, interact mainly through a magnetic dipolar interaction ${ }^{10}$. This classical Coulomb phase is characterized by dipolar spin correlations with a power-law decay ${ }^{12,13}$.

Quantum variants of spin ice ${ }^{14-16}$ have also been found in the magnetic pyrochlore oxides $\mathrm{RE}_{2} \mathrm{TM}_{2} \mathrm{O}_{7}$ (ref. 17) with the rareearth (RE) ions $\mathrm{Yb}$ (refs 18-25) and $\mathrm{Pr}$ (refs 15,16,26,27) and transition-metal (TM) ions such as Ti and Ir, or Sn. In this quantum version of spin ice, the magnetic monopole charges are expected to be carried by fractionalized pseudospin-1/2 quantum-mechanical quasi-particles, spinons, that acquire kinetic energy by hopping via a pseudospin-flip exchange interaction, if they remain deconfined with an energy gap ${ }^{28}$. Thus, these systems provide a unique playground to study the intriguing quantum dynamics of monopolar spinons. Quantum spin ice actually harbours the analogous 'quantum electrodynamics', where the 'electric field' and 'electric charge' describe the Ising magnetic moment and the associated monopole charge, respectively ${ }^{2}$. In particular, $\mathrm{Yb}_{2} \mathrm{Ti}_{2} \mathrm{O}_{7}$ provides a prototypical system described by an effective pseudospin-1/2 quantum spinice model ${ }^{19,20}$, showing strong transverse quantum fluctuations of magnetic dipoles as a result of the following three characteristics: First, the crystal field structure of the $\mathrm{Yb}^{3+}$ ion is characterized by a ground-state Kramers doublet well-separated from the first excited doublet $^{22}$. Second, the Curie-Weiss temperature is weakly ferromagnetic with $\Theta_{\mathrm{CW}} \sim 0.53 \mathrm{~K}$ (ref. 24) as in dipolar spin ice ${ }^{10}$. Third, the $g$-tensor has planar components, $g^{\perp}=4.18$, that are larger than the $\langle 111\rangle$ Ising components $g \|=1.77$ (ref. 22 ), enhancing the contributions to the magnetic neutron scattering cross-sections from planar fluctuations.

An early specific-heat measurement on a powder sample of $\mathrm{Yb}_{2} \mathrm{Ti}_{2} \mathrm{O}_{7}$ revealed a sharp singularity at $0.21 \mathrm{~K}$, signalling a firstorder phase transition ${ }^{21}$. A previous neutron scattering experiment, on a single crystal in which we have subsequently shown that the specific-heat anomaly is reproduced (Supplementary Fig. S1), found an almost collinear ferromagnetic structure with an ordered $\mathrm{Yb}^{3+}$ magnetic moment of $1.1 \mu_{\mathrm{B}}$, a uniform magnetization along [100] and a slow relaxation of the spins over times of the order of $2 \mathrm{~h}$ well below $T_{C} \sim 0.21 \mathrm{~K}$ (ref. 24 ). These results may indicate the formation of an energy gap in the spin excitations below a first-order phase transition to the ferromagnetic state. We have shown from specific-heat measurements (Supplementary Fig. S1) and extended X-ray absorption fine structure (EXAFS) experiments (Supplementary Fig. S2) on three different single crystals (see Methods), that the long-range ferromagnetic order in $\mathrm{Yb}_{2} \mathrm{Ti}_{2} \mathrm{O}_{7}$ is quite sensitive to $\mathrm{Yb}$ deficiency. In particular, our analysis indicates that as the $\mathrm{Yb}$ deficiency increases, the specific-heat anomaly becomes significantly broader and the sharp anomaly is lost (Supplementary Fig. S1). This provides an understanding of the experimental results reported for different single-crystal and polycrystalline samples of $\mathrm{Yb}_{2} \mathrm{Ti}_{2} \mathrm{O}_{7}$, which suggest magnetic correlations remain short-range and dynamic even below $0.21 \mathrm{~K}$ (refs $18,23,25$ ). The variation in the experimental findings on different samples suggests the proximity of this material to a quantum phase transition between a quantum magnetic Coulomb phase (U(1) spin liquid) ${ }^{2}$ and a magnetically ordered phase (Fig. 1a). The magnetic ordering in this case can be understood as a Bose-Einstein condensation of monopolar spinons within the Higgs mechanism ${ }^{29,30}$, which gaps out all the soft spin excitations and takes a first-order character ${ }^{28-30}$, as in the case of normal-to-superconductor transitions ${ }^{31}$. This first-order phase transition at zero
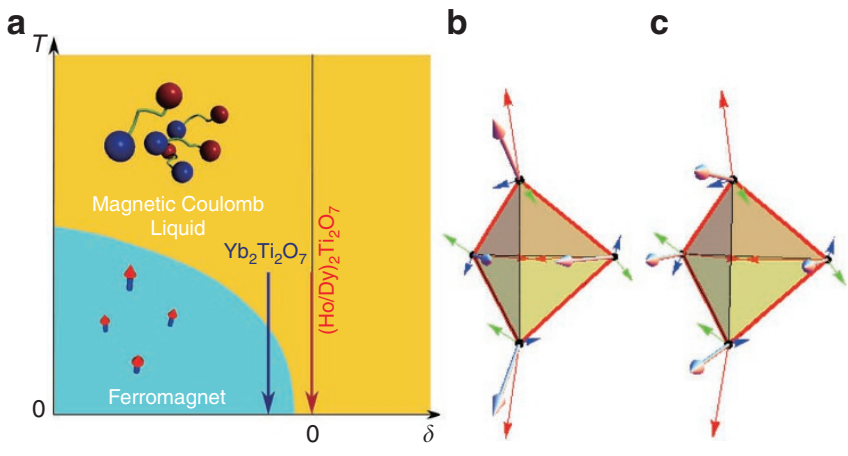

Figure 1 | Schematic phase diagram and hypothetical ordered structures for $\mathbf{Y b}_{\mathbf{2}} \mathbf{T i}_{\mathbf{2}} \mathbf{O}_{\mathbf{7}}$. (a) Schematic phase diagram as a function of temperature $T$ and the relative strength $\delta$ of the $\mathrm{U}(1)$-symmetric planar exchange interaction and the Ising exchange. A first-order Higgs transition appears between a Coulomb liquid phase and a Higgs phase of magnetic monopoles. The other two model parameters $q$ and $K$ in equation (2) are assumed to be negligibly small for dipolar spin ice $(\mathrm{Dy} / \mathrm{Ho})_{2} \mathrm{Ti}_{2} \mathrm{O}_{7}$, whereas they are finite for $\mathrm{Yb}_{2} \mathrm{Ti}_{2} \mathrm{O}_{7}$ as obtained in the present work. Monopoles (blue balls) and antimonopoles (red balls) are illustrated for both the phases. In the magnetic Coulomb liquid phase (yellow), magnetic monopoles are carried by pseudospin- $1 / 2$ fractionalized gapped spinon excitations out of quasi-degenerate spin-ice manifold, obeying a Coulombic law. In the Higgs phase (cyan), monopolar spinons are condensed to form local magnetic dipole moments (arrows) showing ferromagnetic longrange order. $(\mathbf{b}, \mathbf{c})$ Hypothetical ferromagnetically ordered structures of the pseudospins (b) and the magnetic moments

(c) in the low-temperature Higgs phase. The finite planar components of the pseudospins are ascribed to a condensation of monopolar spinons in the $U(1)$ gauge theory ${ }^{2,28}$

temperature should extend to finite temperatures ${ }^{32}$. Suppose that the transition temperature is much lower than an energy $2 J$ required for thermally and dynamically exciting monopolar spinons and that the high-temperature phase does not suffer from a static and thus non-uniform proliferation of monopole charges that causes a spontaneous symmetry breaking. Then, the high-temperature phase may be described as a magnetic Coulomb liquid at temperatures lower than $2 J$, as in dipolar spin ice. Furthermore, the low-temperature phase transition can be effectively described as a Higgs transition of magnetic monopoles, like that of electric monopoles in the classical case $^{33}$. The minimal requirement for the identification of this Higgs transition of magnetic monopoles is the observation of a first-order phase transition separating a high-temperature Coulomb phase that may be characterized by pinch-point features $7,8,12,13$ and a lowtemperature magnetically ordered phase that exhibits finite planar components of pseudospins and gapped spin excitations.

In this article we report evidence of a Higgs transition from a magnetic Coulomb liquid to a ferromagnet, from data that were collected using low-temperature polarized neutron scattering experiments on a single crystal of $\mathrm{Yb}_{2} \mathrm{Ti}_{2} \mathrm{O}_{7}$. We observe that well below $T_{C} \approx 0.21 \mathrm{~K}$ the incident polarized neutron beam is completely depolarized. This is ascribed to the formation of macroscopic ferromagnetic domains. The first-order nature of the phase transition is revealed via the observation of a thermal hysteresis around $T_{C}$. A nearly collinear ferromagnetic structure consistent with previous neutron scattering experiments on the same single crystal ${ }^{24}$ is identified theoretically using an effective pseudospin-1/2 quantum spinice model on a microscopic basis $16,19,20$. The model also reproduces our polarization-dependent magnetic neutron scattering data above $T_{C}$, showing dipolar spin correlations with a remnant of a pinchpoint singularity as a signature of an unusual finite-temperature 

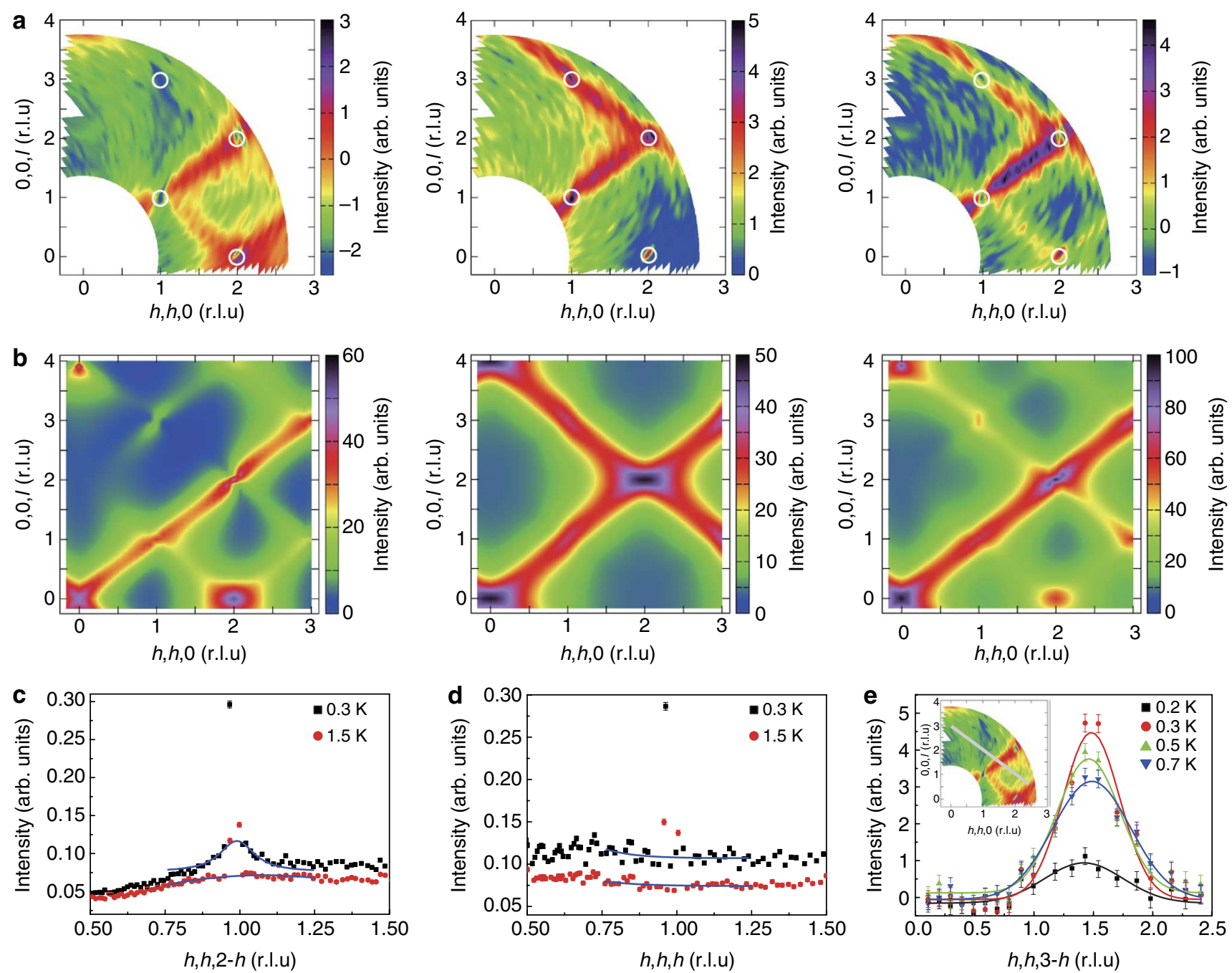

Figure 2 | Magnetic diffuse neutron scattering profiles. The incident neutron spins were polarized along the $Z$ direction. (a) Experimentally observed profiles for the SF (left), NSF (middle) and total (right) magnetic diffuse neutron scattering cross-sections in the $(h, h, l)$ plane above $T_{C}$ at $T=0.3 \mathrm{~K}$. The background levels for the diffuse scattering have been subtracted by making use of the data measured with the incident neutron beam polarized along the $X$ direction parallel to the scattering wavevector $\mathbf{Q}$ (see Supplementary Methods and Supplementary Fig. S3). White circles mark the Bragg spots where the background subtraction scheme is less reliable. The measurements were performed in the energy-integrated diffraction mode up to an energy of $3.64 \mathrm{meV}$. (b) Theoretically calculated magnetic profiles for SF (left), NSF (middle) and total (right) magnetic diffuse neutron scattering cross-sections in the $(h, h, I)$ plane at $T=0.3 \mathrm{~K}$. The profiles were obtained as the equal-time correlators in the RPA (Supplementary Methods) to the pseudospin-1/2 model, $H_{\mathrm{D}}+H_{\mathrm{se}}$. The form factor is not included. (c,d) Neutron scattering intensity for the SF channel along [11-1] and [111] cuts, respectively, through the remnant of the pinch point (111) at $T=0.3$ and $1.5 \mathrm{~K}$. The data were measured with a higher resolution in reciprocal space than the full $(h, h, l)$ profiles. One or two points marking a high level of intensity at and close to the (111) point correspond to the nuclear Bragg peak. The lines are a guide to the eye. (e) Temperature dependence of the total cross-section along [11-1] cut through $(1.5,1.5,1.5)$, as indicated in the inset.

paramagnetic phase, which in practice can be described as a classical magnetic Coulomb liquid $7,8,12,13$.

\section{Results}

Diffuse scattering and pinch-point features above $T_{C}$. Figure $2 \mathrm{a}$ shows the experimental magnetic diffuse neutron scattering crosssections collected at $0.3 \mathrm{~K}$ in the energy-integrated diffraction mode with an integration over the energy transfer up to $3.64 \mathrm{meV}$. The background has been subtracted (Supplementary Methods). Here, the incident neutron spins were polarized along the $Z$ direction, perpendicular to the $(h, h, l)$ plane, allowing us to probe the scattering intensities in the spin-flip (SF) and the non-SF (NSF) channels as well as for the sum of these two channels. A broad intensity from (000) through (111) to (222) is identified in the sum (Fig. 2a, right), as previously reported in unpolarized neutron scattering experiments ${ }^{18}$. This feature is dominant in the NSF channel (Fig. 2a, middle). The [111]-rod scattering is accompanied by a branch from (111) to (220) in the SF channel (Fig. 2a, left), and by another from (222) towards (004) in the NSF channel (Fig. 2a, middle). The anisotropic nature of the spin correlations is clearly seen around (111) in the SF channel (Fig. 2a, left), suggesting a remnant of the pinchpoint singularity ${ }^{13}$ that has been observed as a sharper singularity in dipolar spin ice ${ }^{7,8}$. To examine this structure in more detail, we have performed similar measurements with a higher resolution at 0.3 and $1.5 \mathrm{~K}$. Figure $2 \mathrm{c}$, d show the neutron scattering cross-sections for the SF channel along [11-1] and [111] cuts, respectively, through the (111) point. Whereas the intensity along [111] is featureless and simply increases in magnitude on cooling, the intensity along [11 - 1] clearly shows a growth of the correlation length. This provides direct evidence of the remnant of the pinch-point singularity. 
These polarization-dependent anisotropic neutron scattering results are reproduced theoretically as follows. The $\mathrm{Yb}^{3+} 4 f$ magnetic moment at a site $\mathbf{r}$ is described with the ionic Kramers doublet $^{22}$ and thus the pseudospin-1/2 operator $\hat{\mathbf{S}}_{\mathbf{r}}=\left(\hat{S}_{r}^{x}, \hat{S}_{r}^{y}, \hat{S}_{r}^{z}\right)$ as $\hat{\mathbf{m}}_{\mathbf{r}}=\mu_{\mathrm{B}}\left(g_{\perp}\left(\hat{S}_{r}^{x} \mathbf{e}_{r}^{x}+\hat{S}_{r}^{y} \mathbf{e}_{\mathbf{r}}^{y}\right)+g^{\|} \hat{S}_{r}^{z} \mathbf{e}_{\mathbf{r}}^{z}\right)$ with the locally defined coordinate frame $\left(\mathbf{e}_{\mathbf{r}}^{x}, \mathbf{e}_{\mathbf{r}}^{y}, \mathbf{e}_{\mathbf{r}}^{z}\right.$ ) (Supplementary Methods), where the $Z$ axis points along the $\langle 111\rangle$ direction. The effective Hamiltonian consists of the magnetic dipolar interaction,

$$
H_{\mathrm{D}}=\frac{\mu_{0}}{4 \pi} \sum_{\left\langle\mathbf{r}, \mathbf{r}^{\prime}\right\rangle}\left[\frac{\hat{\mathbf{m}}_{\mathbf{r}} \cdot \hat{\mathbf{m}}_{\mathbf{r}^{\prime}}}{(\Delta r)^{3}}-3 \frac{\left(\hat{\mathbf{m}}_{\mathbf{r}} \cdot \Delta \mathbf{r}\right)\left(\Delta \mathbf{r} \cdot \hat{\mathbf{m}}_{\mathbf{r}^{\prime}}\right)}{(\Delta r)^{5}}\right],
$$

with $\Delta r=|\Delta \mathbf{r}|, \quad \Delta \mathbf{r}=\mathbf{r}-\mathbf{r}^{\prime}$ and a nearest-neighbour anisotropic superexchange interaction ${ }^{16,19}$,

$$
\begin{aligned}
H_{\mathrm{se}}= & \frac{J}{2} \sum_{\left\langle\mathbf{r}, \mathbf{r}^{\prime}\right\rangle}^{\mathrm{n} . \mathrm{n} .}\left[\hat{S}_{r}^{z} \hat{S}_{r^{\prime}}^{z}+\delta \hat{S}_{r}^{+} \hat{S}_{r^{\prime}}^{-}+q e^{2 i \phi_{r, r^{\prime}}} \hat{S}_{r}^{+} \hat{S}_{r^{\prime}}^{+}\right. \\
& \left.+K e^{i \phi_{r, r^{\prime}}}\left(\hat{S}_{r}^{z} \hat{S}_{r^{\prime}}^{+}+\hat{S}_{r}^{+} \hat{S}_{r^{\prime}}^{z}\right)\right]+ \text { h.c. }
\end{aligned}
$$

with the bond-dependent phase factor $\phi_{r, r^{\prime}}$ (Supplementary Methods). Here, $J$ is the coupling constant for the Ising interaction found in spin-ice systems ${ }^{10,25}$, whereas the dimensionless parameters $\delta / q$ and $K$ represent the relative coupling constants of the planar quantum exchange interaction that respect/break the $\mathrm{U}(1)$ pseudospin symmetry and of another interaction that breaks the $2 \pi$ rotation symmetry of the pseudospins about the $\langle 111\rangle$ direction. Figure $2 \mathrm{~b}$ shows the magnetic neutron scattering cross-sections calculated at $0.3 \mathrm{~K}$ within the random phase approximation $(\mathrm{RPA})^{34}$. A set of four adjustable parameters $(J, \delta, q, K)=(0.68 \mathrm{~K},-0$. $8,0.2,-1.0)$ reproduces the experimental profiles shown in Fig. 2a. (Any change of $\delta, q$ or $K$ by \pm 0.1 gives worse results.) The signs and ratios of the coupling constants obtained from the fitting are comparable to those obtained microscopically with a reasonable ratio $V_{p f \pi} /$ $V_{p f \sigma}=0.2$ of the Slater-Koster parameters ${ }^{19}$. The values of $(\delta, q, K)$ are also nearly within the error bars of the recent spin-wave analysis of high-field inelastic neutron scattering results ${ }^{20}$ (Supplementary Methods). The positive coupling constant $J$ for the Ising interaction favours the 2-in, 2-out spin-ice manifold. (Note that the amplitude of $J$ could be underestimated here because of an overestimation of the onset temperature for the instability in the RPA.) Accordingly, a macroscopic number of states are nearly degenerate, in spite of the additional interactions. In the theoretical calculations, the intensity and the sharpness of a remnant of the pinch-point singularity increase with decreasing temperature in the high-temperature paramagnetic phase (Supplementary Fig. S4), in qualitative agreement with our experimental results. These observations support the scenario that the high-temperature phase of $\mathrm{Yb}_{2} \mathrm{Ti}_{2} \mathrm{O}_{7}$ in the temperature range $T_{C}<T<2 J$ is well described as a classical magnetic Coulomb liquid, which hosts the pinch-point features and monopolar spinons that are deconfined with a finite excitation energy.

First-order transition to a ferromagnetic state. In Fig. 2e we present the temperature dependence in the sum of the SF and NSF magnetic neutron scattering cross-sections across the [111] rod through $(1.5,1.5,1.5)$. The broad intensity increases gradually on cooling from 0.7 to $0.3 \mathrm{~K}$, but falls abruptly at $0.2 \mathrm{~K}$, signalling a sudden change in the spin correlations. Actually, the neutron scattering profile at $0.04 \mathrm{~K}$ (Supplementary Fig. S3d) clearly shows that the [111]-rod scattering loses intensity. We have measured the (111) Bragg peak intensity as a function of temperature on warming and cooling, with the incident neutron spins polarized along the $X$ direction. Figure 3 a shows the dependence of the sum of the SF and NSF channels for small angular deviations from the wavevector (111) (rocking-curve scans) both above and below $T_{C}$ with the data collected on warming the sample. By subtracting the $1 \mathrm{~K}$ data, the magnetic components can be clearly observed below $T_{C}$ (Fig. 3b). The integrated intensities for the SF and NSF channels (Fig. 3c) and for the sum of the two (Fig. 3d) show a remarkable hysteresis around $T_{C}$, indicating a first-order magnetic phase transition. It is possible that the magnetic scattering intensity along the diffusive [111] rod is removed to form the magnetic Bragg peaks below $T_{C}$. Well above $T_{C}$ the flipping ratio (the ratio of the NSF to SF cross-sections) at (111) gradually decreases on cooling and then falls more rapidly to unity around $T_{C}$ (Fig. 3e). This indicates that the incident neutron spins are already partially depolarized in the high-temperature phase because of short-range ferromagnetic correlations, and are fully depolarized well below $T_{C}$, pointing to the emergence of macroscopic ferromagnetic domains. Furthermore, the first-order nature of the transition has also been confirmed by a sharp anomaly in the specific heat of the sample, (Supplementary Fig. S1), which closely resembles the earlier result ${ }^{21}$.

The emergent ferromagnetic order without translational symmetry breaking is also understood theoretically by the mean-field analysis (Supplementary Methods) of the same model at $T=0$ with the values of the dimensionless coupling constants $(\delta, q, K)$ determined above. It is found that the mean-field ground state has a noncoplanar pseudospin order with the Ising component $\left\langle S_{r}^{z}\right\rangle \sim 0.43$ and the planar component $\sqrt{\left\langle S_{r}^{x}\right\rangle^{2}+\left\langle S_{r}^{y}\right\rangle^{2}} \sim 0.25$, as shown in Fig. 1b. This is translated to a non-coplanar ferromagnetic order with a slight tilting from the [100] direction by $\sim 1^{\circ}$ and a moment amplitude $1.3 \mu_{\mathrm{B}}$ (Fig. 1c), in fair agreement with the previous neutron diffraction results on the same single crystal ${ }^{24}$. These results on the direction and the amplitude of the magnetic moments also show reasonable agreements with previous Mössbauer results on a powder sample ${ }^{23}$. In this low-temperature phase, no soft spin excitations exist because of the absence of a continuous symmetry in the original model. This slightly non-coplanar ferromagnetic ground state does not alter with $(\delta, q, K)$ within the accuracy of our fitting procedure.

\section{Discussion}

From the viewpoint of gauge theor ${ }^{2,28}$, the high-temperature phase of our system is effectively described as a Coulomb phase, where bosonic fractionalized quasi-particles, 'spinons', carrying magnetic monopole charges are deconfined without a static non-uniform proliferation of monopole charges and host fluctuating U(1) gauge fields responsible for the analogous 'quantum electrodynamics'. These magnetic monopolar spinons can undergo a Bose-Einstein condensation $^{32}$ to form the classical ferromagnetic moment ${ }^{28}$ (Fig. 1a). This pins the global U(1) phase of the spinon fields, and breaks the nontrivial emergent $\mathrm{U}(1)$ gauge structure. This condensation of matter (spinon) fields coupled to gauge fields occurs through the Higgs mechanism ${ }^{29}$. Thus, the ferromagnetic state can be viewed as a Higgs phase of magnetic monopoles.

Our observations support a novel picture that magnetic monopole charges are carried by fractionalized bosonic quasiparticles in the high-temperature magnetic Coulomb liquid. At present, the disappearance of the low-energy gapless excitation spectrum, unique to the Higgs phase, is only suggested by the long magnetic relaxation times seen in this and earlier studies ${ }^{24}$, and must be confirmed by future experiments, which could also identify the spectrum of gapped spin wave excitations having the role of 'Higgs bosons'. It will also be fascinating to search for the quantum phase transition between the magnetic Coulomb phase and the Higgs phase with proper control of the material by pressure or chemical substitution.

\section{Methods}

Single-crystal growth. Single crystals of $\mathrm{Yb}_{2} \mathrm{Ti}_{2} \mathrm{O} 7$, which were also used in the previous published work ${ }^{24}$, were prepared by the floating zone method. Stoichiometric quantities of $\mathrm{Yb}_{2} \mathrm{O}_{3}$ and $\mathrm{TiO}_{2}$ powder were mixed, pressed into rods and 
a

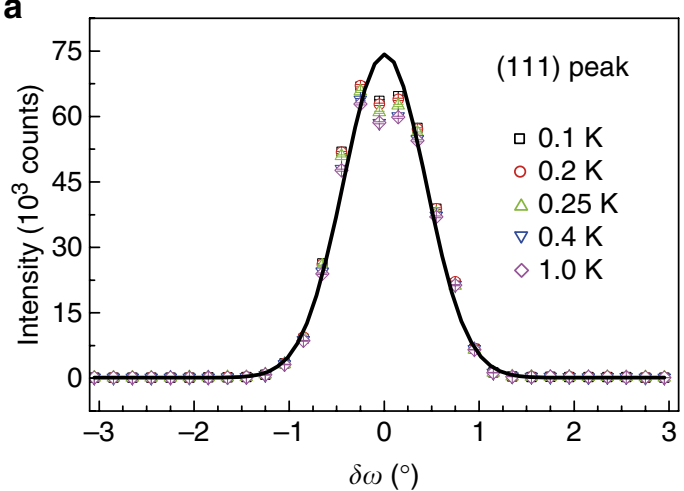

b

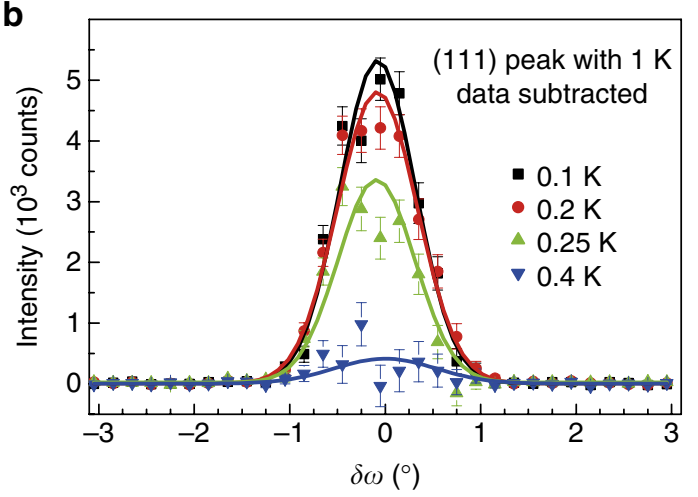

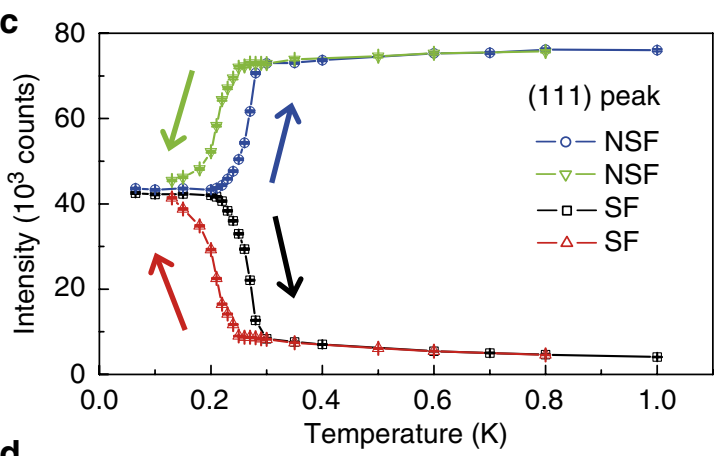

d

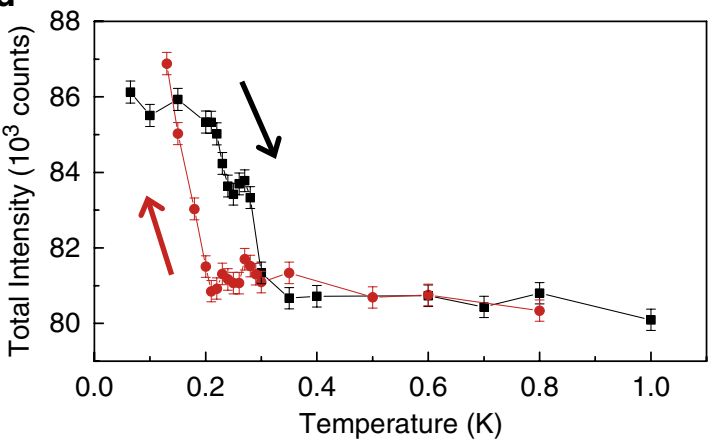

e

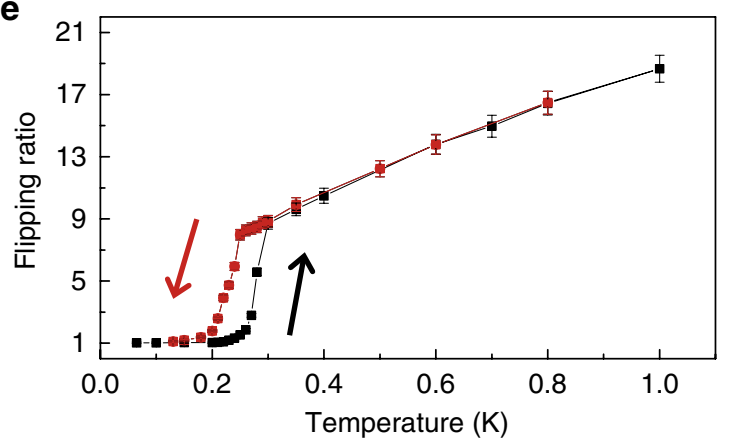

Figure 3 | Hysteresis in the Bragg peak intensity and flipping ratio at (111). The data were collected on cooling and warming across $T_{C}$ in the $X$-direction polarized neutron scattering experiments. To ensure that the system was in thermal equilibrium, measurements well below $T_{C}$ were performed after waiting longer than the magnetic relaxation time, which is of the order of $2 \mathrm{~h}$ at $0.03 \mathrm{~K}$ (ref. 24). (a) The rocking-curve scans of the (111) Bragg peak for the sum of the SF and NSF channels measured while warming the sample. The horizontal axis represents the deviation of the wavevector from (111) with their amplitude being unchanged. The lines are a guide to the eye. All the peaks are instrument resolution limited. (b) The magnetic contribution to the (111) Bragg peak is obtained by subtracting the data measured at $1 \mathrm{~K}$, which is well above $T_{C}$, in the warming sequence. (c) The growth of the integrated intensity for the (111) Bragg peak begins abruptly below $T_{C}$ with a discernible hysteresis in both the SF and the NSF channels. (d) The sum of the SF and the NSF (111) Bragg peak intensities versus temperature showing a hysteresis with temperature. The sum increases by almost $6 \%$ below $T_{C}$ in agreement with the previous measurements ${ }^{24}$. (e) The flipping ratio of the neutron spins at the (111) Bragg peak as a function of temperature. This ratio falls off steeply to unity below $T_{C}$ with a clear hysteresis. The data were collected by first warming and then cooling. This sequence is indicated by the arrows in (c-e).

sintered at $1,150^{\circ} \mathrm{C}$ for $24 \mathrm{~h}$. Using these rods, single crystals were grown in air at a rate of $1.5 \mathrm{~mm} / \mathrm{h}$. The crystals had a typical diameter of $\sim 6 \mathrm{~mm}$ and a length of $20 \mathrm{~mm}$. Powder X-ray diffraction measurements on a pulverized part of the single crystal showed no appreciable amount of any impurity phase.

For comparison with other experimental work that reported the absence of long-range magnetic order, the different methods of sample preparation are worth mentioning. Only three reports are available in the literature. Gardner et al. ${ }^{25}$ synthesized polycrystalline samples of $\mathrm{Yb}_{2} \mathrm{Ti}_{2} \mathrm{O}_{7}$ by firing stoichiometric amounts of $\mathrm{Yb}_{2} \mathrm{O}_{3}$ and $\mathrm{TiO}_{2}$ at $1,350^{\circ} \mathrm{C}$ for several days, with the quality checked by X-ray diffraction. Ross et al. ${ }^{18}$ synthesized single crystals of $\mathrm{Yb}_{2} \mathrm{Ti}_{2} \mathrm{O}_{7}$ using the same floating zone method as our work, but in 4 bars of oxygen and at a faster growth rate of $5 \mathrm{~mm} / \mathrm{h}$. Single crystals prepared by Yaouanc et al. ${ }^{35}$ were also grown by the floating zone method but at an even faster growth rate of $8 \mathrm{~mm} / \mathrm{h}$ in air with and without a subsequent heat treatment for $24 \mathrm{~h}$ at $1,100^{\circ} \mathrm{C}$ under oxygen flow.

Characterization of different single-crystal samples. Here, we discuss the controversy over the presence or absence of a first-order phase transition in $\mathrm{Yb}_{2} \mathrm{Ti}_{2} \mathrm{O}_{7}$ We then demonstrate from measurements of the specific heat and the EXAFS that the quality of the samples significantly alters the low-temperature behaviour of this material. In particular, we show that the long-range ferromagnetic order realized through the first-order phase transition in the single-crystal sample reported in the main text is easily removed by reducing the $\mathrm{Yb}$ content within the sample.

First, let us briefly summarize the previous experimental reports that catalogue the strong sample dependence of the physical properties of $\mathrm{Yb}_{2} \mathrm{Ti}_{2} \mathrm{O}_{7}$. The CurieWeiss temperature, $\Theta_{C W}$, varies considerably from sample to sample. $\Theta_{\mathrm{CW}} \sim 0.53 \mathrm{~K}$ for our sample reported on in the main text and in ref. $24,0.40 \mathrm{~K}$ in ref. $21,0.59 \mathrm{~K}$ in ref. 36 and $0.75 \mathrm{~K}$ in ref. 22. A significant sample dependence is also observed in the low-temperature specific heat in both powder and single-crystal $\mathrm{Yb}_{2} \mathrm{Ti}_{2} \mathrm{O}_{7}$ samples $^{35,37}$. In addition, according to Gardner et al. ${ }^{25}$, a magnetic Bragg peak was observed at (111) with polarized neutron scattering measurements on polycrystalline samples, although neutron spin depolarization was not observed and static ferromagnetic order was ruled out.

In an attempt to rationalize these conflicting reports, we have studied three single-crystal samples of $\mathrm{Yb}_{2} \mathrm{Ti}_{2} \mathrm{O}_{7}$ using specific heat and EXAFS measurements. Sample A was prepared using the method described above and was the sample used for both our polarized neutron scattering measurements and the previous unpolarized measurements ${ }^{24}$. Sample B was produced from a rod sintered at $1,350^{\circ} \mathrm{C}$ rather than $1,150^{\circ} \mathrm{C}$, and was grown in air using a faster growth rate of 
$5 \mathrm{~mm} / \mathrm{h}$ (cf. Ross et al. ${ }^{18}$ who also used a faster growth rate but grew in 4 bars oxygen). Sample C was grown from another rod, which was prepared in the same manner as sample A. However, the molten zone was less stable during the crystal growth.

Supplementary Figure S1 shows the temperature variation of the specific heat for these three samples. Sample A has a sharp singularity at $0.214 \mathrm{~K}$. This is consistent with the observation made in our neutron scattering experiments of the first-order phase transition to a ferromagnetic state. Similar sharp anomalies were also observed for polycrystalline samples in both the early and recent measurements ${ }^{21,37}$. For sample B, the specific-heat anomaly is broadened significantly, signalling the disappearance of the first-order phase transition. The temperature dependence of the specific heat of sample B closely resembles those of the single crystals $^{35,37}$ used for unpolarized neutron scattering experiments ${ }^{18,37,38}$. In sample $\mathrm{C}$, the peak in the heat capacity disappears completely.

The crystal structure of these three samples has been examined by EXAFS at the $\mathrm{Yb} L_{3}$-edge. Supplementary Figure S2 shows the radial distribution functions of these samples extracted from the Fourier transform of EXAFS oscillations, shown in the upper inset. The oscillations become increasingly damped for sample A (black), B (red) and C (green), respectively, indicating either a higher level of disorder or the appearance of more vacancies for sample A through to sample $\mathrm{C}$. The Fourier-transformed radial distribution functions favours the vacancy scenario. Near-edge spectra reveal only the $\mathrm{Yb}^{3+}$ charge state (see the middle inset). More oxygen vacancies, particularly at the $\mathrm{O}(2)$ site located at the centres of $\mathrm{Yb}_{4} \mathrm{O}$ tetrahedra, are created than $\mathrm{Yb}$ and Ti vacancies in samples $\mathrm{B}$ and $\mathrm{C}$. These observations suggest vacancies of all three elements are created cooperatively to maintain charge neutrality. Comparing the EXAFS data with the specific heat curves, one can conclude that the higher the quality of the sample, that is the closer the sample is to stoichiometry, the sharper the magnetic transition. In particular, the low-temperature ferromagnetism discussed in the main text is intrinsic to the most ideal single crystals such as sample A.

Polarized neutron scattering experiments. Measurements were performed at the high-flux polarized diffuse neutron scattering spectrometer DNS, FRM II (Garching, Germany). A ${ }^{3} \mathrm{He} /{ }^{4} \mathrm{He}$ dilution refrigerator insert with an Oxford Instruments cryostat was used for the experimental temperatures from 0.04 to $1 \mathrm{~K}$. A neutron wavelength of $4.74 \AA$ was chosen for all the experiments. The [1-10] direction of the crystals was aligned perpendicular to the horizontal scattering plane so that the $(h, h, l)$ reciprocal plane can be mapped out by rotating the sample. The neutron polarization at the sample position was aligned along the [1-10] direction of the sample, that is the $Z$-direction of the chosen experimental coordinate system (Z-direction polarized neutron scattering). Within this setup, the SF and NSF scattering cross-sections are given by

$$
\begin{gathered}
\left(\frac{\mathrm{d} \sigma}{\mathrm{d} \Omega}\right)^{Z-\mathrm{SF}}=M_{\perp Y}^{*} M_{\perp Y}+\frac{2}{3} I_{\mathrm{SI}}, \\
\left(\frac{\mathrm{d} \sigma}{\mathrm{d} \Omega}\right)^{Z-\mathrm{NSF}}=M_{\perp Z}^{*} M_{\perp Z}+N^{*} N+\frac{1}{3} I_{\mathrm{SI}},
\end{gathered}
$$

respectively, where $M_{\perp Y}^{*} M_{\perp Y}$ and $M_{\perp Z}^{*} M_{\perp Z}$ are the components of the magnetic scattering cross-section in and out of the $(h, h, l)$ scattering plane, respectively, with $Y$ being perpendicular to the scattering wavevector $\mathbf{Q}$. $I_{\mathrm{SI}}$ is the total nuclear spin incoherent scattering cross-section, and $N^{\star} N$ is the nuclear coherent scattering cross-section. In general, the magnetic scattering cross-sections $M_{\perp Y}^{*} M_{\perp Y}$ and $M_{\perp Z}^{*} M_{\perp Z}$ are not identical. Therefore, they can provide information on the anisotropy of the magnetic correlations present in the system. To confirm the observed magnetic scattering, $X$-direction-polarized neutron scattering has also been carried out. In this polarized neutron scattering setup, the neutron polarization is parallel to the scattering vector $\mathbf{Q}$, and can be used to separate the magnetic scattering contribution to be mapped in the SF channel, and nuclear contribution in the NSF channel;

$$
\begin{gathered}
\left(\frac{\mathrm{d} \sigma}{\mathrm{d} \Omega}\right)^{X-\mathrm{SF}}=M_{\perp Y}^{*} M_{\perp Y}+M_{\perp Z}^{*} M_{\perp Z}+\frac{2}{3} I_{\mathrm{SI}}, \\
\left(\frac{\mathrm{d} \sigma}{\mathrm{d} \Omega}\right)^{X-\mathrm{NSF}}=N^{*} N+\frac{1}{3} I_{\mathrm{SI}} .
\end{gathered}
$$

For details, see refs 39,40 .

\section{References}

1. Dirac, P. A. M. Quantised singularities in the electromagnetic field. Proc. R. Soc. A 133, 60-72 (1931).
2. Hermele, M., Fisher, M. P. A. \& Balents, L. Pyrochlore photons: the $U(1)$ spin liquid in a $S=1 / 2$ three-dimensional frustrated magnet. Phys. Rev. B 69, 064404 (2004)

3. Castelnovo, C., Moessner, R. \& Sondhi, S. L. Magnetic monopoles in spin ice. Nature 451, 42-45 (2008).

4. Nagaosa, N., Sinova, J., Onoda, S., MacDonald, A. \& Ong, N. P. Anomalous Hall effect. Rev. Mod. Phys. 82, 1539-1592 (2010).

5. Qi, X.- L., Li, R., Zang, J. \& Zhang, S.- C. Inducing a magnetic monopole with topological surface states. Science 323, 1184-1187 (2009).

6. Sakakibara, T., Tayama, T., Hiroi, Z., Matsuhira, K. \& Takagi, S. Observation of a liquid-gas type transition in the pyrochlore spin ice compound $\mathrm{Dy}_{2} \mathrm{Ti}_{2} \mathrm{O}_{7}$ in a magnetic field. Phys. Rev. Lett. 90, 207205 (2003).

7. Morris, D. J. P. et al. Dirac strings and magnetic monopoles in the spin ice $\mathrm{Dy}_{2} \mathrm{Ti}_{2} \mathrm{O}_{7}$. Science 326, 411-414 (2009).

8. Fennell, T. et al. Magnetic Coulomb phase in the spin ice $\mathrm{Ho}_{2} \mathrm{Ti}_{2} \mathrm{O}_{7}$. Science 326, 415-417 (2009).

9. Giblin, S. R. et al. Creation and measurement of long-lived magnetic monopole currents in spin ice. Nat. Phys. 7, 252-258 (2011).

10. Bramwell, S. T. \& Gingras, M. J. P. Spin ice state in frustrated magnetic pyrochlore materials. Science 294, 1495-1501 (2001).

11. Moessner, R. \& Ramirez, A. Geometrical frustration. Phys. Today 59, 24-29 (2006)

12. Isakov, S. V., Gregor, K., Moessner, R. \& Sondhi, S. L. Dipolar spin correlations in classical pyrochlore magnets. Phys. Rev. Lett. 93, 167204 (2004).

13. Henley, C. L. Power-law spin correlations in pyrochlore antiferromagnets. Phys. Rev. B 71, 014424 (2005).

14. Molavian, H. \& Gingras, M. J. P. Dynamically induced frustration as a route to a quantum spin ice state in $\mathrm{Tb}_{2} \mathrm{Ti}_{2} \mathrm{O}_{7}$ via virtual crystal field excitations and quantum many-body effects. Phys. Rev. Lett. 98, 157204 (2007).

15. Onoda, S. \& Tanaka, Y. Quantum melting of spin ice: emergent cooperative quadrupole and chirality. Phys. Rev. Lett. 105, 047201 (2010).

16. Onoda, S. \& Tanaka, Y. Quantum fluctuations in the effective pseudospin-1/2 model for magnetic pyrochlore oxides. Phys. Rev. B 83, 094411 (2011).

17. Gardner, J. S., Gingras, M. J. P. \& Greedan, J. E. Magnetic pyrochlore oxides. Rev. Mod. Phys. 82, 53-107 (2010).

18. Ross, K. A et al. Two-dimensional kagome correlations and field induced order in the ferromagnetic XY pyrochlore $\mathrm{Yb}_{2} \mathrm{Ti}_{2} \mathrm{O}_{7}$. Phys. Rev. Lett. 103, 227202 (2009).

19. Onoda, S. Effective quantum pseudospin-1/2 model for $\mathrm{Yb}$ pyrochlore oxides. J. Phys: Conf. Series. 320, 012065 (2011).

20. Ross, K. A. et al. Quantum excitations in quantum spin ice. Phys. Rev. X 1, 021002 (2011).

21. Blöte, W. J., Wielinga, R. F. \& Huiskamp, W. J. Heat-capacity measurements on rare-earth double oxides $\mathrm{R}_{2} \mathrm{M}_{2} \mathrm{O}_{7}$. Physica 43, 549-568 (1969).

22. Hodges, J. A., Bonville, P., Forget, A., Rams, M., Królas, K. \& Dhalenne, G. The crystal field and exchange interactions in $\mathrm{Yb}_{2} \mathrm{Ti}_{2} \mathrm{O}_{7}$. J. Phys: Condens. Matter 13, 9301-9310 (2001).

23. Hodges, J. A. et al. First-order transition in the spin dynamics of geometrically frustrated $\mathrm{Yb}_{2} \mathrm{Ti}_{2} \mathrm{O}_{7}$. Phys. Rev. Lett. 88, 077204 (2002).

24. Yasui, Y. et al. Ferromagnetic transition of pyrochlore compound $\mathrm{Yb}_{2} \mathrm{Ti}_{2} \mathrm{O}_{7}$. J. Phys. Soc. Jpn 72, 3014-3015 (2003).

25. Gardner, J., Ehlers, G., Rosov, N., Erwin, R. W. \& Pertovic, C. Spin-spin correlations in $\mathrm{Yb}_{2} \mathrm{Ti}_{2} \mathrm{O}_{7}$ : a polarized neutron scattering study. Phys. Rev. B 70, 180404(R) (2004).

26. Zhou, H. D. et al. Dynamic spin ice: $\mathrm{Pr}_{2} \mathrm{Sn}_{2} \mathrm{O}_{7}$. Phys. Rev. Lett. 101, 227204 (2008).

27. Machida, Y., Nakatsuji, S., Onoda, S., Tayama, T. \& Sakakibara, T. Timereversal symmetry breaking and spontaneous Hall effect without magnetic dipole order. Nature 463, 210-213 (2010).

28. Savary, L. \& Balents, L. Coulombic quantum liquids in spin- $1 / 2$ pyrochlores. Phys. Rev. Lett. 108, 037202 (2012).

29. Fradkin, E. \& Shenker, S. H. Phase diagrams of lattice gauge theories with Higgs fields. Phys. Rev. D. 19, 3682-3697 (1979).

30. Rokhsar, D. S. \& Kivelson, S. A. Superconductivity and the quantum hard-core dimer gas. Phys. Rev. Lett. 61, 2376-2379 (1988).

31. Anderson, P. W. Plasmons, gauge invariance, and mass. Phys. Rev. 130, 439-442 (1963).

32. Banerjee, A., Isakov, S. V., Damle, K. \& Kim, Y. B. Unusual liquid state of hardcore bosons on the pyrochlore lattice. Phys. Rev. Lett. 100, 047208 (2008).

33. Powell, S. Higgs transitions of spin ice. Phys. Rev. B 84, 094437 (2011).

34. Kao, Y.- J., Enjalran, M., Del Maestro, A., Molavian, H. R. \& Gingras, M. J. P. Understanding paramagnetic spin correlations in the spin-liquid pyrochlore $\mathrm{Tb}_{2} \mathrm{Ti}_{2} \mathrm{O}_{7}$. Phys. Rev. B 68, 172407 (2003).

35. Yaouanc, A., Dalmas de Réotier, P., Marin, C. \& Glazkov, V. Single-crystal versus polycrystalline samples of magnetically frustrated $\mathrm{Yb}_{2} \mathrm{Ti}_{2} \mathrm{O}_{7}$ : specific heat results. Phys. Rev. B 84, 172408 (2011).

36. Bramwell, S. T., Field, M. N., Harris, M. J. \& Parkin, I. P. Bulk magnetization of the heavy rare earth titanate pyrochlores - a series of model frustrated magnets. J. Phys: Condens. Matter 12, 483-495 (2000). 
37. Ross, K. A. et al. Dimensional evolution of spin correlations in the magnetic pyrochlore $\mathrm{Yb}_{2} \mathrm{Ti}_{2} \mathrm{O}_{7}$. Phys. Rev. B 84, 174442 (2011).

38. Bonville, $\mathrm{P}$ et al. Transitions and spin dynamics at very low temperature in the pyrochlores $\mathrm{Yb}_{2} \mathrm{Ti}_{2} \mathrm{O}_{7}$ and $\mathrm{Gd}_{2} \mathrm{Sn}_{2} \mathrm{O}_{7}$. Hyperfine. Interact. 156-157, 103 (2004).

39. Schweika, W. XYZ-polarisation analysis of diffuse magnetic neutron scattering from single crystals. J. Phys: Conf. Series 211, 012026 (2010).

40. Schärpf, O. \& Capellmann, H. The XYZ-difference method with polarised neutrons and the separation of coherent, spin incoherent, and magnetic scattering cross sections in a multidetector. Phys. Status Solidi. A 135, 359-379 (1993).

\section{Acknowledgements}

We thank J.S. Gardner, B.D. Gaulin and A. Yaouanc for discussions. We thank Harald Schneider for technical supports in the neutron scattering experiments. We acknowledge Chih-Wen Pao and other staff of BL07A of the NSRRC who helped perform the EXAFS measurements. This work is partially supported by National Science Council, Taiwan, under grants no. NSC 96-2739-M-213-001, NSC 99-2112M-007-020 (L.J.C.), NSC 100-2112-M-002 -013 -MY3, NSC-99-2120-M-002-005 (Y.J.K.), by NTU under grant no. 10R80909-4 (Y.J.K.), and by grants-in-aid for Scientific Research under grant no. 21740275 and no. 24740253 from Japan Society for the Promotion of Science (S.O.) and under grant no. 19052006 (S.O.) and no. 19052004 (K.K.) from the Ministry of Education, Culture, Sports, Science and Technology, Japan.

\section{Author contributions}

L.J.C. performed the neutron scattering experiments and wrote the manuscript; S.O. and Y.J.K. performed the theoretical calculations and wrote the manuscript; Y.S. designed and performed the neutron scattering experiments; Y.Y. grew the single-crystal sample; K.K. and M.R.L. supervised the neutron scattering experiments. M.R.L. carried out the heat capacity measurements. K.D.T. performed the EXAFS studies. All the authors discussed the results and the manuscript.

\section{Additional information}

Supplementary Information accompanies this paper at http://www.nature.com/ naturecommunications

Competing financial interests: The authors declare no competing financial interests.

Reprints and permission information is available online at http://npg.nature.com/ reprintsandpermissions/

How to cite this article: L-J Chang et al. Higgs transition from a magnetic Coulomb liquid to a ferromagnet in $\mathrm{Yb}_{2} \mathrm{Ti}_{2} \mathrm{O}_{7}$. Nat. Commun. 3:992 doi: 10.1038/ncomms1989 (2012).

License: This work is licensed under a Creative Commons Attribution-NonCommercialShare Alike 3.0 Unported License. To view a copy of this license, visit http:// creativecommons.org/licenses/by-nc-sa/3.0/ 